\title{
Experimental violation of a Bell's inequality in time with weak measurement
}

\author{
Agustin Palacios-Laloy ${ }^{1}$, François Mallet ${ }^{1}$, François Nguyen ${ }^{1}$, \\ Patrice Bertet $^{1 *}$, Denis Vion ${ }^{1}$, Daniel Esteve ${ }^{1}$, and Alexander Korotkov ${ }^{2}$ \\ ${ }^{1}$ Quantronics Group, Service de Physique de l'État Condensé (CNRS URA 2464), \\ DSM/IRAMIS/SPEC, CEA-Saclay, \\ 91191 Gif-sur-Yvette Cedex, France and \\ ${ }^{2}$ Deparment of Electrical Engineering, \\ University of California, Riverside, CA 92521-0204, USA
}

\begin{abstract}
The violation of J. Bell's inequality with two entangled and spatially separated quantum twolevel systems (TLS) is often considered as the most prominent demonstration that nature does not obey "local realism". Under different but related assumptions of "macrorealism", plausible for macroscopic systems, Leggett and Garg derived a similar inequality for a single degree of freedom undergoing coherent oscillations and being measured at successive times. Such a "Bell's inequality in time", which should be violated by a quantum TLS, is tested here. In this work, the TLS is a superconducting quantum circuit whose Rabi oscillations are continuously driven while it is continuously and weakly measured. The time correlations present at the detector output agree with quantum-mechanical predictions and violate the inequality by 5 standard deviations.
\end{abstract}

PACS numbers: $74.50,03.65,82.25$ 


\section{Introduction}

The violation of J. Bell's inequality [1, 2] is the most prominent example of a situation where the predictions of quantum mechanics are incompatible with a large class of classical theories. In the early 1980s, Aspect and coworkers [3] brought an experimental proof of this violation using pairs of spatially-separated polarization-entangled photons. By demonstrating an excess of correlations between the polarizations measured on the two photons of a pair, they ruled out descriptions of nature satisfying the very general conditions known as local realism. This striking finding also contributed to transform the so-called quantum weirdness into a useful resource for information processing. Shortly after, quantum cryptography protocols and quantum algorithms exploiting entanglement were indeed proposed [4]. Following a reasoning similar to that of Bell, Leggett and Garg derived in 1985 an inequality that can be seen as a "Bell's inequality in time", which applies to any single macroscopic system measured at successive times [5] and fullfiling the assumptions of macrorealism: (A1) the system is always in one of its macroscopically distinguishable states, and (A2) this state can be measured in a non invasive way, i.e. without perturbing the subsequent dynamics of the system. Quantum mechanics however contradicts both assumptions, which can lead to an excess of correlations between subsequent measurements and to a violation of this inequality. Ruskov and coworkers [6] then adapted the inequality to the situation where a two-level systems (TLS) is continuously and weakly monitored during its coherent oscillations. Using such a weak monitoring, we report here an experimental test of a Bell's inequality in time (see also the recent works [7, 8]), yielding results in excellent agreement with simple quantum-mechanical predictions and in contradiction with a large class of macrorealistic models.

\section{Bell's inequalities in space and in time}

We start by briefly recalling the experimental protocol of the usual CHSH test [2] of Bell's inequalities (see Fig. 1a). It consists in identically preparing many times a pair of quantum TLS in a maximally entangled state such as $\left|\psi^{-}\right\rangle=(|\uparrow \downarrow\rangle-|\downarrow \uparrow\rangle) / \sqrt{2}$. Each member of the pair is then distributed to two observers $\mathrm{A}$ and $\mathrm{B}$, who perform projective

measurements of the TLS spin $\sigma_{i}^{A, B}= \pm 1$ along one of two directions $a_{i}(i=1,2)$ for A and 

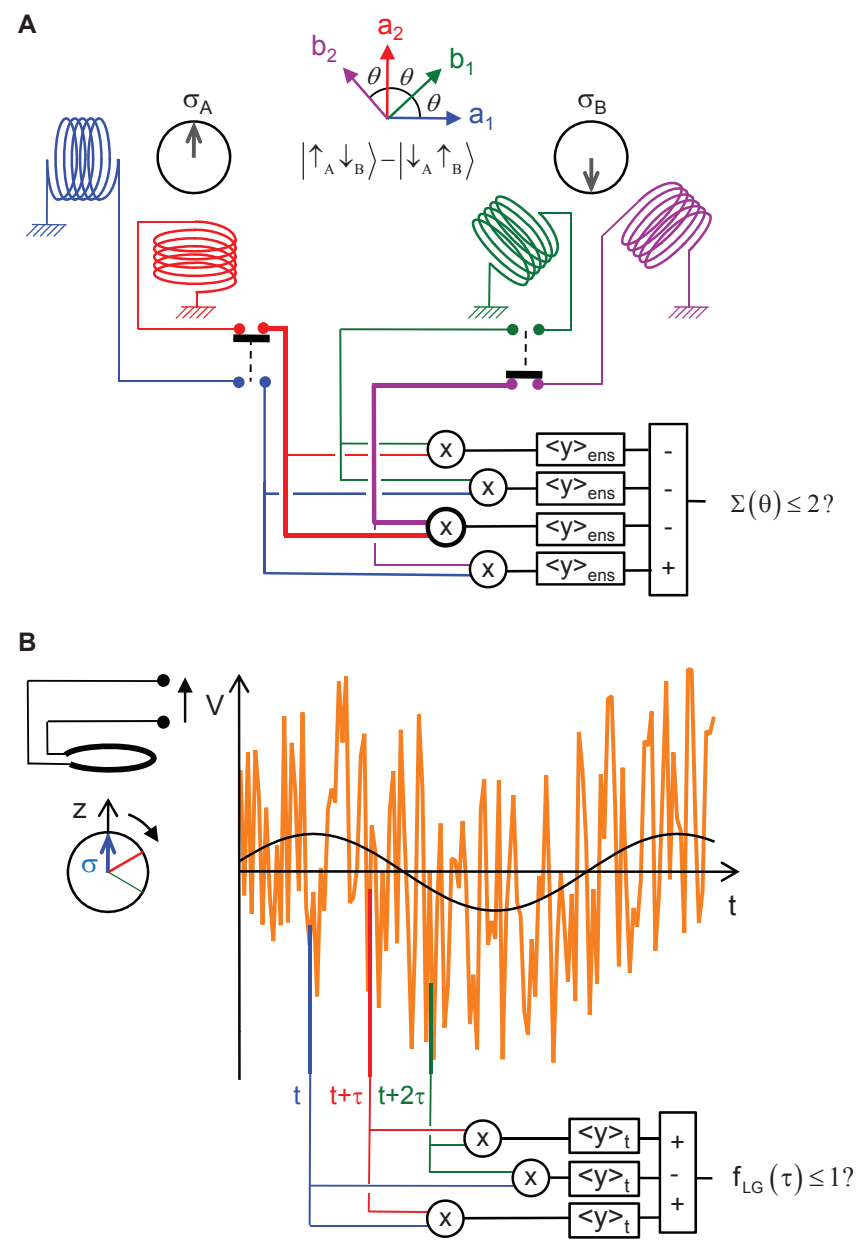

Figure 1: Comparison between two thought experiments which test the usual CHSH Bell's inequality and the Bell's inequality in time. (A) CHSH inequality: two maximally entangled spins $\sigma_{A}$ and $\sigma_{B}$ are sent to two spatially separated observers A and B. Each of the observers measures with pick-up coils his spin along one of two possible directions ( $a_{1}$ and $a_{2}$ for $\mathrm{A}$, and $b_{1}$ and $b_{2}$ for $\mathrm{B}$ ); the four directions make angles $\theta$ as depicted. By repeating this experiment on a statistical ensemble, a linear combination $\Sigma$ of the four possible correlators between measurements on a spin pair is computed. Local realism requires $-2 \leq \Sigma \leq 2$, while quantum mechanics predicts $\Sigma=2 \sqrt{2}$ for $\theta=45^{\circ}$. (B) Bell's inequality in time with weak measurement: a single spin $\sigma$ undergoing coherent oscillations at frequency $\omega_{R}$ is continuously measured with a pick-up coil coupled to it so weakly that the time for a complete projective measurement would be much longer than the period of oscillations $T_{R}=2 \pi / \omega_{R}$. From the noisy time trace recorded in the steady state, one computes a linear combination $f_{L G}$ of the three time-averaged-correlators between the readout outcomes at three times separated by $\tau$. Macrorealism requires $f_{L G} \leq 1$ for any $\tau$, while quantum mechanics predicts $f_{L G}=1.5$ at $\tau=T_{R} / 6$. 
$b_{i}$ for $\mathrm{B}$, with these directions forming angles $\left(a_{1}, b_{1}\right)=\left(b_{1}, a_{2}\right)=\left(a_{2}, b_{2}\right) \equiv \theta$ as shown in Fig. 17. The two observers then combine all their measurements to compute the Bell sum $\Sigma(\theta)=-K_{11}+K_{12}-K_{22}-K_{21}$ of the correlators $K_{i j}(\theta)=\left\langle\sigma_{i}^{A} \sigma_{j}^{B}\right\rangle$. The Bell's theorem, based on a simple statistical argument, states that according to all local realistic theories

$$
-2 \leq \Sigma(\theta) \leq 2
$$

However, standard quantum mechanics predicts that this inequality is violated, with a maximum violation $\Sigma(\theta=\pi / 4)=2 \sqrt{2}$. Many experimental tests, and in particular those performed by A. Aspect [3] have verified this violation [11, 12].

While quantum entanglement between two spatially separated TLS is at the heart of the previous violation, Leggett and Garg proposed a similar inequality [5] holding for a single degree of freedom $-1 \leq z(t) \leq 1$ fulfilling the assumptions of macrorealism $(\mathrm{z}(\mathrm{t})$ defined at any time, and measurable with no perturbation). Using a simple arithmetic argument à la Bell, they showed that

$$
z\left(t_{0}\right) z\left(t_{1}\right)+z\left(t_{1}\right) z\left(t_{2}\right)-z\left(t_{0}\right) z\left(t_{2}\right) \leq 1
$$

for all $\left\{t_{i}\right\}$. Consequently, an observer measuring $z$ on many identical systems, either at $t_{0}$ and $t_{1}=t_{0}+\tau$, or at $t_{0}$ and $t_{2}=t_{0}+2 \tau$, or at $t_{1}$ and $t_{2}$ should find ensemble-averaged correlators $K_{i j}\left(t_{0}, \tau\right)=\left\langle z\left(t_{i}\right) z\left(t_{j}\right)\right\rangle$ (for $i, j=0,1,2$, with $i<j$ ) satisfying the LeggettGarg's inequality:

$$
f_{L G}\left(t_{0}, \tau\right) \equiv K_{01}+K_{12}-K_{02} \leq 1
$$

Quantum mechanics on the other hand predicts that, applied to the case of a quantum TLS undergoing coherent oscillations at frequency $\omega_{R}$, this inequality is violated for wellchosen values of $\tau$, with maximum violation $f_{L G}\left(t_{0}, \tau=\pi / 3 \omega_{R}\right)=1.5$ independent of $t_{0}$. Here, the delay $\tau$ between successive measurements plays the role of the angle $\theta$ between the measurement directions in the Bell's inequality (1), justifying the nickname "Bell's inequality in time". The excess of correlations predicted by quantum mechanics, compared to the macrorealistic case, can be interpreted as resulting from the projection of the TLS state on a $\sigma_{z}$ eigenstate induced by the first measurement.

As shown in [6], the very same conclusions also hold if the TLS undergoing coherent oscillations is continuously and weakly monitored along $\sigma_{z}$ (see Fig. 1B) instead of being 
projectively measured at well-defined times. The detector now delivers an output signal $V(t)=(\delta V / 2) z(t)+\xi(t)$ proportional to $z(t)$ with some additional noise $\xi(t)$. Macrorealism implies that the dynamics of the system at time $t+\tau$ is fully uncorrelated with the detector noise at time $t$ so that $\langle\xi(t) z(t+\tau)\rangle_{t}=0$. The detector's output correlation function $K(\tau)=\langle V(t) V(t+\tau)\rangle_{t} /(\delta V / 2)^{2}$ is then simply equal to $\langle z(t) z(t+\tau)\rangle_{t}$. By averaging inequality (2) over $t_{0}$ in the steady-state, the Bell's inequality in time (3) becomes

$$
f_{L G}(\tau) \equiv 2 K(\tau)-K(2 \tau) \leq 1
$$

and should be violated by a quantum TLS in the very same way as discussed above. Here the violation is however not due to a strong projection of the TLS wavefunction induced by measurements at well-defined times of its evolution, but rather to the continuous partial projection caused by the measurement during the TLS coherent evolution, which reinforces correlations between the detector output at successive times.

\section{Experimental setup}

Our experimental setup (see Fig. 2A and supplementary information A for probing inequality (4) closely implements the proposal discussed above, making use of the possibilities offered by the so-called circuit quantum electrodynamics (circuit-QED) architecture [13, 14] where a superconducting artificial TLS is coupled to a superconducting coplanar waveguide resonator. The TLS consists here of the two lowest energy states $g$ and $e$ of a modified

Cooper-pair box of the transmon type [17, 18]. These two states can be regarded as "macroscopically distinguishable" because the dipole moment of the $g-e$ transition is of the order of $10^{4}$ atomic units. On the other hand, the only degree of freedom of this system is the phase difference between the superconducting order parameters on both sides of the Josephson junction forming the Cooper-pair box, conjugate to the number of Cooper pairs passed through the junction; this phase is a collective variable whose degree of macroscopicity is still under debate [15, 16].

The TLS transition frequency is $\omega_{g e} / 2 \pi=5.304 \mathrm{GHz}$, below the resonance frequency $\omega_{c} / 2 \pi=5.796 \mathrm{GHz}$ of the resonator to which it is capacitively coupled for its measurement. Two microwave sources $V_{d}$ and $V_{m}$ drive and measure the TLS at frequencies $\omega_{g e}$ and $\omega_{c}$, respectively. In order to continuously monitor the induced Rabi oscillations up to a few 

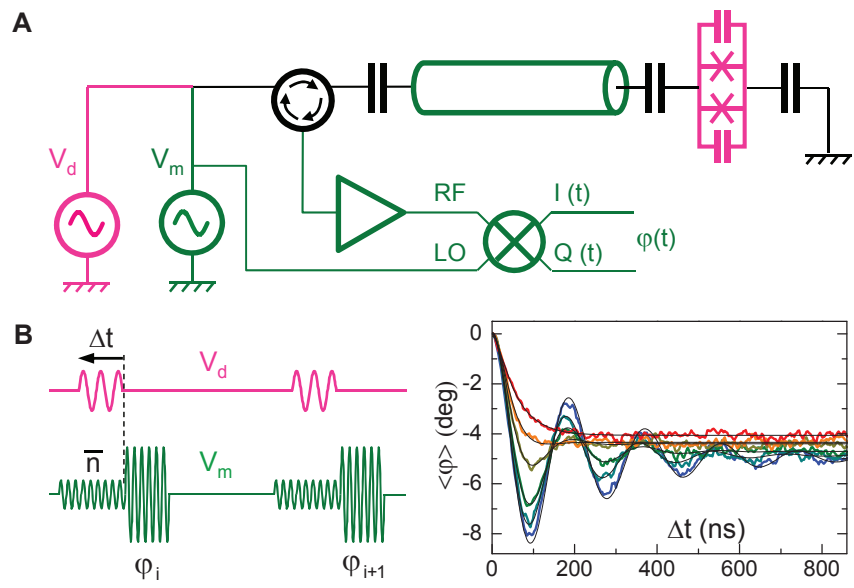

Figure 2: (A) Experimental implementation of the thought experiment in Fig. 11B) with a quantum electrical circuit. The spin (or TLS) is a Cooper pair box of the transmon type (magenta) capacitively coupled to a microwave resonator (cavity sketched as a green coaxial cable). Two microwave sources $V_{d}$ and $V_{m}$ are used to drive and measure the transmon at $\omega_{g e}$ and $\omega_{c}$, respectively. The reflected microwave at $\omega_{c}$ is routed through a circulator to a cryogenic amplifier followed by an I-Q demodulator. The time dependent phase $\varphi(t)$ measured by the demodulator carries the information about the TLS state. (B) Measurement-induced dephasing of the TLS as a function of the measurement strength, i.e. the amplitude of $V_{m}$ or the mean photon number $\bar{n}$ in the cavity. Measured ensemble-averaged Rabi oscillations $\langle\varphi(\Delta t)\rangle$ in presence of $\bar{n}=0,1,2,5,10$ and 20 photons (from blue to red curve), showing the transition from weak to strong measurement. Each curve is fitted to a solution of Bloch equations (thin black lines), in quantitative agreement with expected measurement-induced dephasing rates (see online supplementary information D).

tens of $\mathrm{MHz}$, we implement a resonator bandwidth of $\kappa / 2 \pi=30.3 \pm 0.8 \mathrm{MHz}$ (quality factor $191 \pm 5$ ) by designing the appropriate resonator input capacitance [14]. With these parameters, the TLS is sufficiently detuned from the resonator for their interaction to be well described by the so-called dispersive Hamiltonian $\hat{H}=\hbar \chi \hat{n} \hat{\sigma}_{z}$ [13], with $\hat{n}$ the photon number inside the readout mode and $\chi$ the dispersive coupling constant. The resonator frequency is thus shifted by $\pm \chi / 2 \pi= \pm 1.75[-0.11 /+0.14] \mathrm{MHz}$ depending on the TLS state (see supplementary information $\mathrm{B}$ ). The phase $\varphi$ of a microwave signal at $\omega_{c}$ therefore acquires a TLS state-dependent shift after being reflected by the resonator, and provides a non-destructive readout of the TLS as demonstrated in numerous experiments [13, 14, 19]. 
In our setup, the reflected signal is routed through a circulator to a cryogenic amplifier and is then measured by homodyne detection at room temperature, yielding the two field quadratures $I(t)$ and $Q(t)$. These time traces provide a continuous measurement of the TLS with a strength proportional to the signal input power, and thus to the intra-resonator average photon number $\bar{n}$. In a fully quantum-mechanical description of the measurement process using the quantum trajectory formalism [19], each quadrature can be written $X(t)=$ $\bar{X}+(\delta X / 2)\left\langle\hat{\sigma}_{z}\right\rangle_{c}(t)+\xi_{0}(t)$, where $\left\langle\hat{\sigma}_{z}\right\rangle_{c}(t)$ is the expectation value of $\hat{\sigma}_{z}$ conditioned on the whole history of the detector outcome $X\left(t^{\prime}\right)$ for $t^{\prime} \leq t, \delta X$ is the maximum detector signal proportional to the measurement signal amplitude $\sqrt{\bar{n}}$, and $\xi_{0}(t)$ is the total output noise of the amplifier. Our test of inequality (4) consists in accurately measuring the steady state value of $K(\tau)=\langle(X(t)-\bar{X})(X(t+\tau)-\bar{X})\rangle_{t} /(\delta X / 2)^{2}$ with a low measuring power, while the TLS is coherently driven.

\section{Measurement-induced-dephasing in ensemble averaged Rabi oscillations}

Recent experiments have already investigated the back-action of the measurement on a TLS with a similar circuit-QED setup [20]. However only ensemble averaged quantities (i.e. obtained by averaging the outcomes of many identical experimental sequences) had been considered prior to this work, and the only detectable effect of a measurement on the TLS dynamics was some extra dephasing, as demonstrated in [20] by measuring the broadening of the TLS resonance line in presence of a field in the resonator. This measurement back-action results from the dependence of the TLS frequency $\omega_{g e}(t)=\omega_{g e}+2 \chi n(t)$ on the photon number $n(t)$ stored in the resonator: fluctuations of $n$ around $\bar{n}$ cause dephasing with a rate $\Gamma_{\phi}^{p h}(\bar{n})=8 \bar{n} \chi^{2} / \kappa$ proportional to the measurement strength. We first perform a series of control measurements to verify on ensemble-averaged Rabi oscillations our quantitative understanding of the measurement-induced dephasing. After a field of $\bar{n}$ photons (see supplementary information $\mathrm{C}$ ) is established inside the resonator using $V_{m}$, a Rabi pulse of duration $\Delta t$ is applied to the TLS with $V_{d}$ (see Fig. 2 B), followed by a strong measurement pulse. The phase $\langle\varphi(\Delta t)\rangle$ of the reflected measurement pulse is averaged over an ensemble of typically $10^{4}$ identical experimental sequences, yielding the data displayed in Fig. 2 B. One observes that for sufficiently low measurement strength $\bar{n}$, the coherent dynamics is only weakly affected by the measurement. This is the regime where the Bell's inequality in time 
can be tested. For stronger measurement strength, the oscillations are progressively washed out and replaced by an exponential damping. For even stronger measurement strengths, the characteristic time of the exponential becomes longer and longer (see Fig. 2B), revealing that a strong measurement inhibits the transition of the TLS from ground to excited state as expected from the quantum Zeno effect [21, 23]. We checked that these data are in quantitative agreement with the expected measurement-induced dephasing (see supplementary information D). However, it is important to realize that this set of measurements would be unchanged if our driven quantum TLS was replaced by a precessing classical spin, such as a macroscopic ferromagnet. Indeed, a macrospin obeys similar equations of motion as the expectation value of the spin of a TLS, namely Bloch equations. Thus no conclusion about the correlations between measurements at different times can be drawn that would allow a test of inequality (41).

\section{Continuous measurement of Rabi oscillations in the frequency domain}

We measure these correlations by monitoring the system in its steady state, long after the transient ensemble averaged Rabi oscillations such as shown in Fig. 2B have been washed out. Instead of applying microwave pulses, the sources $V_{d}$ and $V_{m}$ are now continuously ON. The quantity of interest is the two-time correlation function $K(\tau)$, whose direct calculation from the measured time traces $X(t)$ is difficult in our setup because the amplifier noise dominates the output signal. However, this added noise can be removed by processing the signal in the frequency domain.

For this purpose, we compute the square modulus $S_{I}$ and $S_{Q}$ of the Fourier transforms of $I(t)$ and $Q(t)$, to obtain the detector output power spectrum $S(\omega)=S_{I}(\omega)+S_{Q}(\omega)$. The signal power spectrum is then obtained by subtracting the amplifier noise spectrum $S_{O F F}(\omega)$ measured when the two sources $V_{d}$ and $V_{m}$ are OFF from the signal-plus-noise spectrum $S_{O N}(\omega)$ measured with both sources $\mathrm{ON}$, and by dividing this difference by the independently measured frequency response $R(\omega)$ of the measuring line (see supplementary information E). Typical curves are shown in Fig. 3A. They show a single peak located at the Rabi frequency (already known from the time-domain measurements), without any harmonics within the $50 \mathrm{MHz}$ detection window. The output spectrum of a continuously monitored TLS undergoing coherent oscillations [27-29] has been the subject of a number 

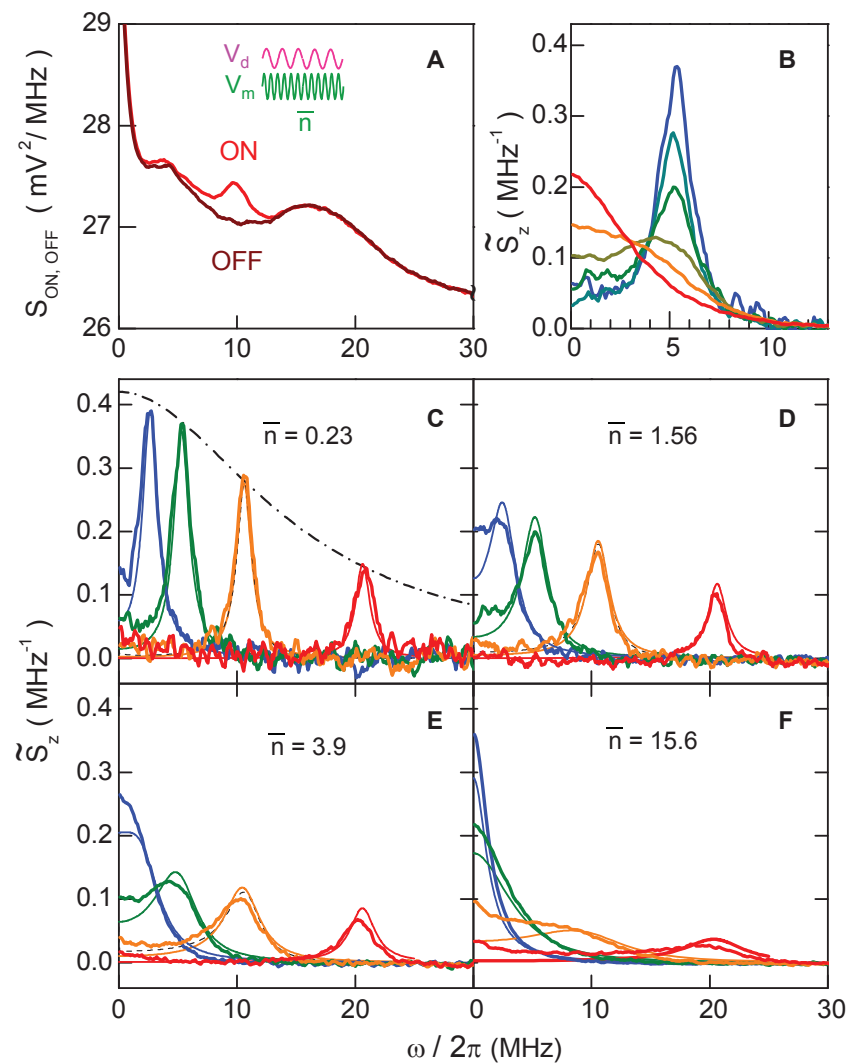

Figure 3: Continuous monitoring of the TLS driven at the Rabi frequency $\omega_{R}$ for different measurement strengths $\bar{n}$. Each power spectrum is acquired in 40 to 80 minutes. (A) Spectral densities $S_{O N}(\omega)$ and $S_{O F F}(\omega)$ when $V_{d}$ and $V_{m}$ are both OFF (brown) or both ON (red); here $\omega_{R} / 2 \pi=10 \mathrm{MHz}$ and $\bar{n}=1$. The difference between ON and OFF shows a peak at the Rabi frequency. (B) Normalized Rabi spectra $\tilde{S}_{z}(\omega)$ after correction from the frequency response of the measuring line and conversion of the output voltage into units of $\sigma_{z}$, at $\omega_{R} / 2 \pi=5 \mathrm{MHz}$ and $\bar{n}=0.23,0.78,1.56,3.9,7.8$, and 15.6 (from blue to red). The curves show the weak to strong measurement transition. (C-F) Normalized Rabi peaks at $\omega_{R} / 2 \pi=2.5,5,10$ and $20 \mathrm{MHz}$ (from blue to red) for $\bar{n}=0.23(\mathrm{C}), 1.56(\mathrm{D}), 3.9(\mathrm{E})$, and $15.6(\mathrm{~F})$. Thick and thin color lines are respectively the experimental spectra and those calculated from a theoretical analytical formula (see text and supplementary information G) using only independently measured parameters (including $\chi / 2 \pi=1.8 \mathrm{MHz})$. Dashed black lines on top of the orange curves in $(\mathrm{C}, \mathrm{D}, \mathrm{E})$ are Rabi peaks obtained by numerical simulation with the same parameters. The dotted-dashed black curve in $\mathrm{C}$ is the Lorentzian frequency response $C(\omega)$ of the resonator. 
of theoretical calculations [9, 24, 25], and precise knowledge of all sample parameters allows us to obtain for the first time a quantitative comparison with these theories. Indeed, we can convert the signal power spectrum in spin units by dividing it by a conversion factor $(\delta V / 2)^{2}$ measured in a calibration experiment by saturating the $g-e$ transition (see supplementary information $\mathrm{F}$ ). The variation of the resulting $\tilde{S}_{z}(\omega)$ spectrum with increasing measurement power is shown in Fig. 3B and is in good agreement with theoretical predictions [9, 24, 25]. These data clearly show the transition from weak to strong measurement in a continuously monitored driven TLS: at low $\bar{n}$, the spectrum consists of a single Lorentzian peak at $\omega_{R}$; upon increasing the measurement strength, the Lorentzian broadens towards low frequencies, and for strong measurements, the spectrum becomes a Lorentzian centered at zero frequency, similar to that of an incoherent TLS jumping stochastically between its two states. In terms of quantum trajectories, the Lorentzian spectra obtained in these two regimes are indirect signatures of the weak measurement-induced quantum phase diffusion along the Rabi trajectory, and of the quantum jumps made by the spin during the strong measurement. The theoretical curves shown in Fig. 3 are obtained using an analytical formula derived from the solution of Bloch equations [26] in which the finite detector bandwidth is taken into account phenomenologically (see supplementary information $\mathrm{G}$ ); the accuracy of this formula was checked by direct numerical integration of the system's master equation (see Fig. $3 \mathrm{C}-\mathrm{E}$ and $(\mathrm{H})$. The agreement between theory and experiment is good for $\bar{n} \leq 5$ but is only qualitative at larger $\bar{n}$, possibly due to a breakdown of the dispersive approximation.

\section{Experimental test of the Bell's inequality in time}

We now turn to the test of inequality (4). We measure a Rabi peak at $\omega_{R} / 2 \pi=10.6 \mathrm{MHz}$ with $\bar{n}=0.78$ photons and a $30 \mathrm{MHz}$ detection window. Under macrorealistic assumptions, the only effect of the bandwidth of the resonator would be to reduce the measured signal by its Lorentzian response function $C(\omega)=1 /\left[1+(2 \omega / \kappa)^{2}\right]$; we thus have to correct for this effect by dividing the measured spectral density $\tilde{S}_{z}(\omega)$ by $C(\omega)$. We then compute $K(\tau)$ by inverse Fourier transform of $S_{z}(\omega)=\widetilde{S}_{z}(\omega) / C(\omega)$. The experimental and theoretical Rabi peaks as well as the corresponding $f_{L G}(\tau)$ curves are plotted in Fig. 4, showing good overall agreement despite residual low-frequency noise possibly originating from low-frequency fluctuations of $\omega_{g e}$. The error bars on $f_{L G}(\tau)$ are the sum of the systematic errors in the calibration of 
$\delta V, \kappa$, and $R(\omega)$, and of the statistical error on the measured spectrum (see supplementary information II).
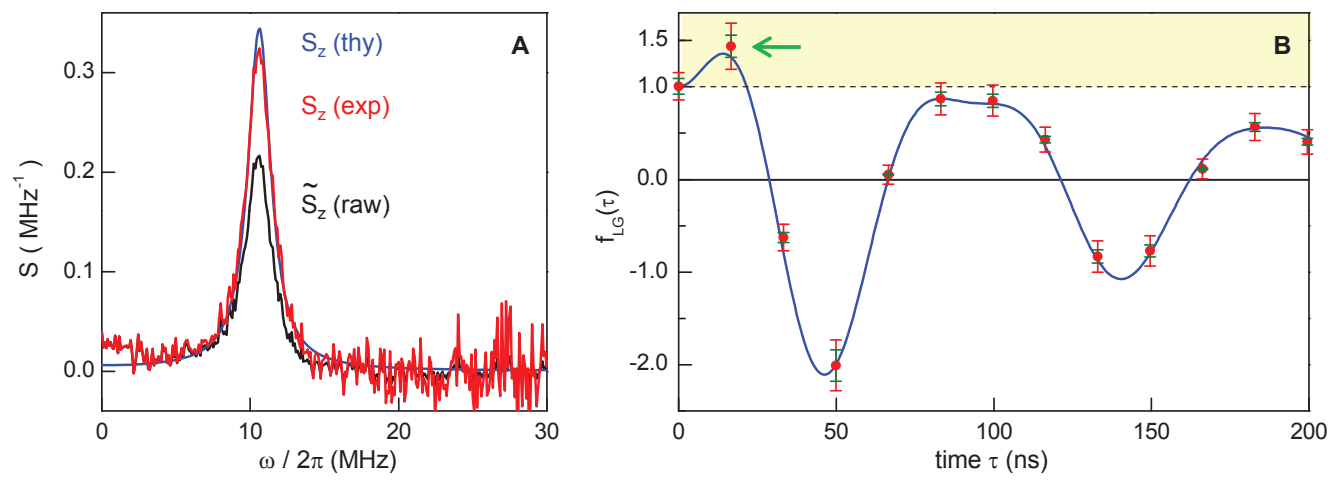

Figure 4: Experimental violation of the "Bell's inequality in time" introduced in Fig. 1B. A) experimental (red) and theoretical (blue) spectral densities $S_{z}$, calculated or measured at $\omega / 2 \pi=$ 10.6 MHz and $\bar{n}=0.78$. The experimental curve is obtained by correcting the raw $\tilde{S}_{z}$ spectrum ( black line), acquired in 13 hours with a $30 \mathrm{MHz}$ bandwidth, from the frequency response $C(\omega)$ of the resonator (see Fig. $3 \mathrm{C}$ ). The blue curve is calculated with $\Gamma_{1}^{-1}=200 \mathrm{~ns}$ and $\Gamma_{2}^{-1}=150 \mathrm{~ns}$ (see supplementary information G). B) experimental (dots) and theoretical (blue line) Leggett-Garg quantity $f_{L G}(\tau)=2 K(\tau)-K(2 \tau)$, with $K(\tau)$ the signal autocorrelation function obtained by inverse Fourier transform of the $S_{z}$ curves in the left panel. Green error bars correspond to the maximum systematic error associated with calibration and $C(\omega)$, whereas red ones also include a two standard deviation wide statistical error $\pm 2 \sigma(\tau)$ associated with the experimental noise on $\tilde{S}_{z}$. The Leggett-Garg inequality is violated (yellow region) at $\tau=17 \mathrm{~ns}$ (see green arrow) by $5 \sigma$.

We first note that we find $K(0)=f_{L G}(0)=1.01 \pm 0.15$, a value close to 1 that directly results from the independent calibration of $\delta V$. Since $K(0)$ represents the variance of $z(t)$ and $|z(t)| \leq 1$, this confirms that at any time $z(t)= \pm 1$ as expected for a quantum TLS. A classical macrospin oscillating as shown in Fig. 3 and calibrated with the same method would have given a variance of $1 / 2$ instead. Note also that $K(0)=1$ could never be deduced from ensemble averaged Rabi oscillations such as those of Fig. 23. This is an example of the specific interest of correlation-function measurements compared to time-domain ensemble averaged signals. Most importantly, we observe that $f_{L G}(\tau)$ goes above the classical limit of 1 , reaching $1.44( \pm 0.12) \pm 2 \times(\sigma=0.065)$ at $\tau=17 \mathrm{~ns} \sim \pi / 3 \omega_{R}$ and thus violating inequality (4) by 5 standard deviations $\sigma$. This maximum of $f_{L G}$, slightly below the ideal 
value of 1.5 in absence of decoherence, is a direct signature of the invasive character of the measurement process, which projects partially but continuously the TLS towards the state corresponding to the detector output. It is the interplay between this continuous projection and the coherent dynamics that yields the violation of the inequality. More quantitatively, the maximum of $f_{L G}$ is in agreement with the quantum prediction of 1.36 when taking into account the independently measured relaxation and dephasing rates of the TLS. This violation of the Leggett-Garg inequality rules out a simple interpretation of $K(\tau)$ as the correlation function of a classical macrospin. It therefore brings further evidence that a collective degree of freedom characterizing a Josephson circuit can behave quantummechanically. It also demonstrates that the back-action of a weak measurement, far from being a simple noise that spoils quantum coherence as could be deduced from ensemble averaged measurements, tends also to reinforce correlations between measurements made at different times.

It is interesting to discuss in what respect the assumptions made in analysing the experimental data influence the final result: apart from simple corrections relying on classical electromagnetism, we determine the main normalization factor $\delta V$ by saturating the TLS transition and assuming that the ensemble averaged spin, either classical or quantum, obeys Bloch equations. We checked this assumption in Fig. 2 and supplementary Fig. 7 , which show in particular that the excursion of the signal when driving the spin is symmetric around the saturation value, as it would be for classical macrospins. The observed violation is thus not an artefact of our analysis framework, and represents more than a mere self-consistency check of a quantum model.

\section{Conclusion}

In conclusion, we have reported the experimental violation of a "Bell's inequality in time" by continuously monitoring the state of a superconducting artificial TLS while it performed Rabi oscillations. The measured two-time correlation function of the detector output reveals strong non-classical correlations between the signal already recorded and the TLS subsequent evolution. Our work thus brings a further proof of the truly quantum-mechanical character of Josephson artificial atoms. It is moreover a first step towards the test of a number of important predictions for such a system [9], and towards certain quantum feedback schemes: 
if the continuous monitoring could be performed with a quantum-limited amplifier [30, 31], it would become indeed possible to stabilize the phase of the Rabi oscillations by feeding back the demodulated signal onto the amplitude or frequency of the source that drives the TLS. This would modify the shape of the Rabi peak, on top of which a narrow line should develop [10]. The correlations demonstrated in this work could then constitute a key resource for quantum feedback, in the same way as entanglement is a resource for quantum information processing.

\section{Acknowledgments}

We acknowledge financial support from European projects EuroSQIP and SCOPE, and from ANR project Quantjo and C'Nano Ile-de-France for the nanofabrication facility at SPEC. We gratefully thank P. Senat, P. Orfila and J.-C. Tack for technical support, and acknowledge useful discussions within the Quantronics group and with A. Lupascu, A. Wallraff and R. Ruskov.

Author contributions: A.K., P.B., \& A.P.L. did the theoretical work, A.P.L., F.M., P.B., D.V., \& D.E. designed the experiment, A.P.L. fabricated the sample, A.P.L., F.M., P.B., \& F.N. performed the measurements, A.P.L., F.M., D.V., \& P.B. analyzed the data, and all the authors contributed to the writing of the manuscript.

Correspondance should be addressed to P.B.

[1] Bell, J.S., On the Einstein Podolvski Rosen Paradox. Physics (N.Y.) 1, 195-200 (1965).

[2] Clauser, J.F., Horne, M.A., Shimony, A., \& Holt, R.A. Proposed Experiment to Test Local Hidden-Variable Theories. Phys. Rev. Lett. 23, 880-884 (1969).

[3] Aspect, A., Grangier, P., \& Roger, G. Experimental Realization of Einstein-Podolsky-RosenBohm Gedankenexperiment: A New Violation of Bell's Inequalities. Phys. Rev. Lett. 49, 91-94 (1982).

[4] Nielsen, M.A. \& Chuang, I.L. Quantum Computation and Quantum Information, Cambridge University Press (2000). 
[5] Leggett, A.J. \& Garg, A. Quantum mechanics versus macroscopic realism: Is the flux there when nobody looks? Phys. Rev. Lett. 54, 857-860 (1985).

[6] Ruskov, R., Korotkov, A.N., \& Mizel, A. Signatures of Quantum Behavior in Single-Qubit Weak Measurements. Phys. Rev. Lett. 96, 200404 (2006).

[7] Goggin, M. E. et al. Violation of the Leggett-Garg inequality with weak measurement of photons. arXiv:0907.1679.

[8] Xu, J.S., Li, C.F., Zou, X.B., \& Guo, G.C. Experimentally identifying the transition from quantum to classical with Leggett-Garg inequalities, arXiv:0907.0176.

[9] Korotkov, A.N. \& Averin, D.V. Continuous weak measurement of quantum coherent oscillations. Phys. Rev. B 64, 165310 (2001).

[10] Ruskov R. \& Korotkov A. N. Quantum feedback control of a solid-state qubit. Phys. Rev. B 66, 041401 (2002).

[11] Ansmann, M. et al. Violation of Bell's inequality in Josephson phase qubits. Nature 461, 504-506 (2009).

[12] Chow, J. M. et al. Entanglement Metrology Using a Joint Readout of Superconducting Qubits. arXiv:0908.1955.

[13] Blais, A., Huang, R., Wallraff, A., Girvin, S. M., \& Schoelkopf, R. J. Cavity quantum electrodynamics for superconducting electrical circuits: An architecture for quantum computation. Phys. Rev. A 69, 062320 (2004).

[14] Wallraff, A. et al. Strong coupling of a single photon to a superconducting qubit using circuit quantum electrodynamics. Nature 431, 162-167 (2004).

[15] Leggett, A. J. Testing the limits of quantum mechanics: motivation, state of play, prospects. J. Phys.: Condens. Matter 14, R415-R451 (2002).

[16] Leggett, A. J. Realism and the physical world. Rep. Prog. Phys. 71, 022001-6 (2008).

[17] Koch, J. et al. Charge-insensitive qubit design derived from the Cooper pair box. Phys. Rev. A 76, 042319 (2007).

[18] Schreier, J.A. Suppressing charge noise decoherence in superconducting charge qubits. et al., Phys. Rev. B 77, 180502 (2008).

[19] Gambetta, J. et al. Quantum trajectory approach to circuit QED: Quantum jumps and the Zeno effect. Phys. Rev. A 77, 012112 (2008).

[20] Schuster, D.I. et al. ac Stark Shift and Dephasing of a Superconducting Qubit Strongly Coupled 
to a Cavity Field. Phys. Rev. Lett. 94, 123602 (2004).

[21] Misra, B. \& Sudarshan, E.C.G. The Zeno's paradox in quantum theory. J. Math. Phys. Sci. 18, 756-763 (1977).

[22] Itano, W. M., Heinzen, D. J., Bollinger, J. J. and Wineland, D. J. Quantum Zeno effect. Phys. Rev. A 41, 2295-2300 (1990).

[23] Bernu, J. et al. Freezing Coherent Field Growth in a Cavity by the Quantum Zeno Effect. Phys. Rev. Lett. 101, 180402 (2008).

[24] Goan, H.S. \& Milburn, G.J. Dynamics of a mesoscopic charge quantum bit under continuous quantum measurement. Phys. Rev. B 64, 235307 (2001).

[25] Shnirman, A., Mozyrsky, D., \& Martin, I. Output spectrum of a measuring device at arbitrary voltage and temperature. Europhys. Lett. 67, 840-846 (2004).

[26] Torrey, H.C. Transient Nutations in Nuclear Magnetic Resonance. Phys. Rev. 76, 1059-1068 (1947).

[27] Il'ichev, E. et al. Continuous Monitoring of Rabi Oscillations in a Josephson Flux Qubit. Phys. Rev. Lett. 91, 097906 (2003).

[28] Deblock, R., Onac, E., Gurevich, L., \& Kouwenhoven, L. P. Detection of Quantum Noise from an Electrically Driven Two-Level System. Science 301, 203-206 (2003).

[29] Manassen, Y., Hamers, R. J., Demuth, J. E., \& Castellano Jr, A. J. Direct observation of the precession of individual paramagnetic spins on oxidized silicon surfaces. Phys. Rev. Lett. 62, 2531-2534 (1989).

[30] Castellanos-Beltran, M. A., Irwin, K. D., Hilton, G.C., Vale L. R., \& Lehnert, K. W. Amplification and squeezing of quantum noise with a tunable Josephson metamaterial. Nature Physics 4, 929-931 (2008).

[31] Bergeal, N. et al. Analog information processing at the quantum limit with a Josephson ring modulator. arXiv:cond-mat/0805.3452. 


\section{Supplementary Information}

\section{A. Sample fabrication, setup, and measurement protocol}

The sample is fabricated using standard lithography techniques. In a first step, a $200 \mathrm{~nm}$ Niobium thin-film is sputtered on a high-resistivity oxidized Silicon substrate. It is patterned with optical lithography followed by reactive ion etching of the Niobium to form the Coplanar Waveguide resonator. The transmon is then patterned by e-beam lithography followed by double-angle evaporation of two Aluminum thin-films, the first one being oxidized to form the junction oxide. The sample is glued on a microwave printed-circuit board, enclosed in a copper box, and thermally anchored to the mixing chamber of a dilution refridgerator at typically $20 \mathrm{mK}$.

Measurement signals are generated by mixing the output of a microwave source $\left(V_{d}\right.$ or $\left.V_{m}\right)$ with DC pulses generated by arbitrary waveform generators, using DC coupled mixers (not shown in Fig. 1). They are then sent to the input microwave line that includes bandpass filters and attenuators at various temperatures $(77 \mathrm{~dB}$ in total $)$. The output line contains a $4-8 \mathrm{GHz}$ bandpass filter, a circulator and two isolators (not shown in Fig. 1), and a cryogenic amplifier CITCRYO1-12 (from Caltech) with $38 \mathrm{~dB}$ gain and noise temperature $T_{N}=4 \mathrm{~K}$. The output signals are further amplified at room-temperature yielding a total gain of $56 \mathrm{~dB}$, and finally mixed down using an I/Q mixer with a synchronized local oscillator at the same frequency. The $I$ and $Q$ quadratures are filtered with a $50 \mathrm{MHz}$ low-pass filter and further amplified with power gain 100 ; the total gains on channels $I$ and $Q$ are equated with a precision better than $0.5 \%$. Both quandratures are then sampled by a fast digitizer and transferred to a computer that processes them.

In the case of ensemble-averaged Rabi oscillations, $200 \mathrm{~ns}$ long measurement pulses are used; the corresponding $X(t)$ traces are averaged over $10^{4}$ identical sequences (repetition rate: $200 \mathrm{kHz}$ ), yielding time traces as shown in Fig. 2B.

In the case of power spectrum measurements, we compute the Fast Fourier Transform $X(\omega)$ on records of $1024 \mathrm{X}(\mathrm{t})$ samples separated by $10 \mathrm{~ns}$. We average $S_{X}=|X(\omega)|^{2}$ over

$10^{5}-10^{6}$ identical sequences, yielding spectra as shown in Fig. 3A. In these experiments, it is crucial to accurately subtract the amplifier noise, whose power is about 60 times stronger than the Rabi peak we want to measure. For that purpose, we measure separately and 


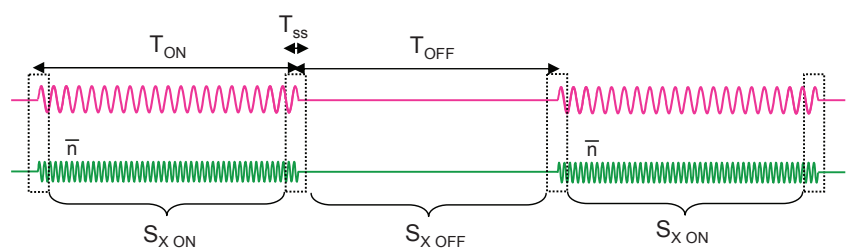

Figure 5: Experimental procedure for measuring the signal power spectra. Both microwave sources $V_{d}$ and $V_{m}$ are switched $\mathrm{ON}$ and $\mathrm{OFF}$ during $T_{O N}=T_{O F F}=2.5 \mathrm{~ms}$. Both quadratures are sampled, their Fast Fourier Transform calculated and squared to compute the power spectra $S_{X} O N$ and $S_{X O F F}$. Dashed rectangles show the $T_{S S}=5 \mu$ s waiting times for establishing the system steady state.

subtract the signal + noise $S_{X O N}$ and the noise $S_{X O F F}$ by alternating periods of duration $T_{O N}=T_{O F F}=2.5 \mathrm{~ms}$, during which both microwave sources $V_{d}$ and $V_{m}$ are ON, or both are OFF. Each time the pulses are switched ON or OFF, we wait a time $T_{s s}=5 \mu$ s to let the system reach its new steady state. The resulting experimental sequence is shown in supplementary Fig. 5. Note that this subtraction also suppresses the influence of long-term drifts of the amplifiers gains.

\section{B. Sample parameters determination}

The resonator parameters $\omega_{c}$ and $\kappa$ given in the text, as well as the TLS frequency $\omega_{g e}$ are determined by standard spectroscopic measurements and are consistent with the design values. In all the experiments with no field in the resonator, the TLS frequency is fixed at $\omega_{g e} / 2 \pi=5.304 \mathrm{GHz}$ by tuning the magnetic field. The $\chi$ value, which is used to calibrate the mean photon number $\bar{n}$, is determined as follows:

When the TLS state changes from $g$ to $e$, the phase of the reflected microwave signal at the resonator frequency varies by $2 \delta \varphi_{0}=4 \arctan (2 \chi / \kappa)$. We thus measure $\delta \varphi_{0}$ by applying a strong and long driving pulse $V_{d}$ that saturates the $g$-e transition and results in an almost equal 50\% population of $g$ and $e$. In this experiment, the resonator is probed continuously with a low-amplitude field $(\bar{n} \sim 2)$ while the reflected signal is measured by heterodyne detection with a local oscillator detuned from $V_{m}$ by $3.2 \mathrm{MHz}$. The resulting beating pattern in the two quadratures $X(t)$ is ensemble averaged over a few $10^{5}$ identical sequences, and its phase $\varphi(t)$ is fitted and plotted in supplementary Fig. 6. The plot yields 


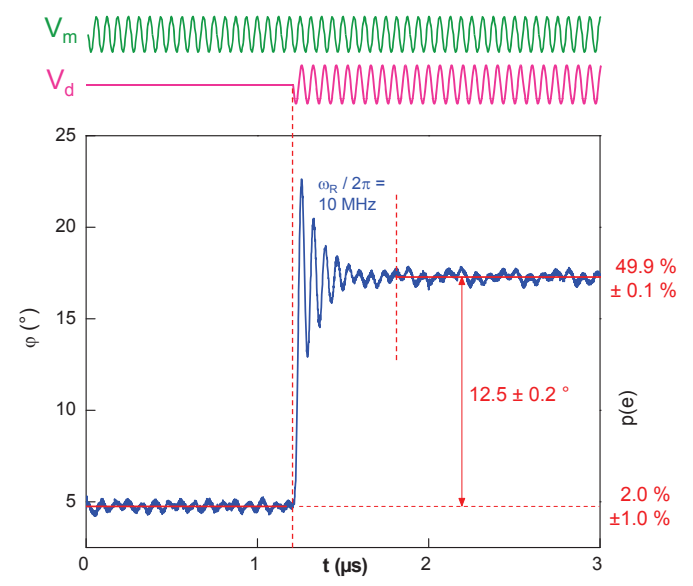

Figure 6: Ensemble averaged measurement of $\varphi(t)$ when a driving pulse saturates the $g-e$ transition starting from $g$. The measurement is continuous with $\bar{n} \sim 2$ photons.

a shift $\delta \phi_{0}=12.5 \pm 0.2^{\circ}$ between no driving and saturation.

For converting this shift into a $\chi$ value, we take into account two effects:

(i) the TLS is not perfectly in its ground state $g$ in the absence of microwave drive due to residual thermal excitation to $e$. We have measured this thermal population $p(e)_{0}=$ $0.02 \pm 0.01$ by measuring the noise spectrum with $V_{d}$ being switched OFF.

(ii) the saturation induced by the driving field is slightly below $50 \%$ due to longitudinal and transversal relaxation. The population $p(e)_{s t}$ in the excited state at saturation is indeed given by the steady state solution to Bloch equations

$$
p(e)_{s t}=\frac{1}{2}-\left[\frac{1}{2}-p(e)_{0}\right] \frac{1+\left(\Gamma_{2}^{-1} \delta \omega\right)^{2}}{1+\left(\Gamma_{2}^{-1} \delta \omega\right)^{2}+\left(\omega_{R}^{2} \Gamma_{1}^{-1} \Gamma_{2}^{-1}\right)} .
$$

Here $\omega_{R} / 2 \pi=10 \mathrm{MHz}$ is fixed by the driving strength, $\Gamma_{1}^{-1}=200 \pm 10 \mathrm{~ns}$ and $\Gamma_{2}^{-1}=$ $150 \pm 10 \mathrm{~ns}$ are independantly measured, and $\delta \omega$ is the residual detuning of the driving source from the TLS resonance. In our experiment the driving frequency was scanned to experimentally maximize $\delta \phi_{0}$ so that $\delta \omega \sim 0$. This yields $p(e)_{s t}=0.496 \pm 0.001$ instead of 0.5 .

Taking (i) and (ii) into account, we thus calculate $\delta \phi_{0}=(0.95 \pm 0.02) \delta \varphi_{0}$. Maximizing all the uncertainties including those on $\kappa$, we finally obtain $\chi / 2 \pi=\kappa \tan \left(\delta \varphi_{0} / 2\right) / 4 \pi=$ $1.75(-0.11 /+0.14) \mathrm{MHz}$, also consistent with the TLS parameters determined by spectroscopy. 


\section{Calibration of the mean photon number $\bar{n}$ at frequency $\omega_{c}$}

The calibration of $\bar{n}$ as a function of the applied input power $P$ of $V_{m}$ is needed for checking that the measurement-induced dephasing is quantitatively understood (see D) and for producing the theoretical curves of Figs. 3 and 4 without any fitting parameters. To perform this calibration, we measure the AC Stark shift- $2 \chi \bar{n}$ of the TLS frequency $\omega_{g e}$ as a function of the input power $P$; $\chi$ being known $(\operatorname{se}(\mathrm{B})$, the shift provides an in-situ calibration of $\bar{n}(P)$.

Note that all the curves presented in this article were obtained with the TLS driving source $V_{d}$ tuned in resonance with the AC Stark shifted TLS frequency. For that, the TLS spectroscopy peak in presence of the very same $\bar{n}$ field was always measured just before each acquisition.

\section{Measurement-induced dephasing and Quantum Zeno Effect}

Since measurement-induced dephasing is a key ingredient of the present work, we checked our quantitative understanding of it. For that, we measure ensemble-averaged Rabi oscillations (see supplementary Fig. 7) in presence of a perturbing field of $\bar{n}$ photons at frequency $\omega_{c}$, which mimicks the effect of a continuous measuring field. In this experiment, the AC stark shifted TLS frequency $\omega_{g e}(t)=\omega_{g e}-2 \chi n(t)$ fluctuates with the number of photons $n(t)$, which fluctuates because of the shot noise in the measurement signal. In an ensemble-averaged viewpoint, this leads to pure dephasing of the TLS with a dephasing rate $\Gamma_{\phi}^{p h}(\bar{n})=8 \bar{n} \chi^{2} / \kappa$ already observed in other experiments [20]. Now, because Rabi oscillations at $\omega_{R}$ are most sensitive to the noise spectral density at $\omega_{R}[\mathrm{~S} 3]$ and because the shot noise in the measurement signal is filtered by the resonator response $C(\omega)=1 /\left[1+(2 \omega / \kappa)^{2}\right]$, one expects for the Rabi oscillations a pure dephasing rate

$$
\Gamma_{\phi}^{p h}\left(\omega_{R}, \bar{n}\right)=\Gamma_{\phi}^{p h}(\bar{n}) C\left(\omega_{R}\right) .
$$

Supplementary Fig. 7 confirms all these expectations: the Rabi oscillations are progressively washed out and replaced by an exponential damping when the measurement strength $\bar{n}$ is increased. For even stronger measurements (larger $\bar{n}$ ), the damping time constant increases (see Fig. 7B), indicating an inhibition of the TLS transition from $g$ to $e$ that is a 

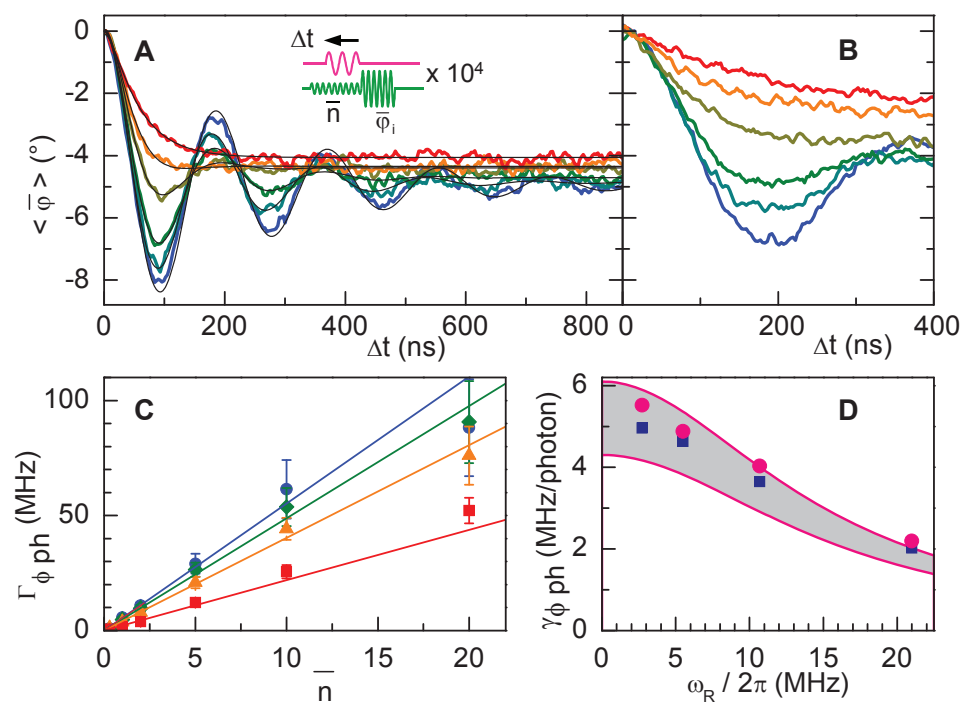

Figure 7: Determination of the measurement-induced dephasing using ensemble-averaged Rabi oscillations recorded with the protocol of Fig. 2B. (A) Same data as in Fig. $2 \mathrm{~B}$ with $\omega_{R} / 2 \pi=5 \mathrm{MHz}$ and $\bar{n}=0,1,2,5,10$ and 20 (blue to red). Fits to the solution of Bloch equations (thin black lines) yield the measurement-induced dephasing rate $\Gamma_{\phi}^{p h}$, after subtraction of other decoherence contributions deduced from the curve at $\bar{n}=0$. (B) Similar experimental data at $\omega_{R} / 2 \pi=2.5 \mathrm{MHz}$ and same photon numbers. When the measurement strength is increased, the time dependence at short times changes from quadratic to approximately linear with an increasing time constant, which is a manifestation of the Quantum Zeno effect. (C) Experimental values (dots with error bars) of $\Gamma_{\phi}^{p h}(\bar{n})$ for $\omega_{R} / 2 \pi=2.5,5,10$ and $20 \mathrm{MHz}$ (blue to red). Solid lines are linear fits of the data taken at $\bar{n} \leq 5$, yielding the measurement-induced dephasing rate per photon $\gamma_{\phi}^{p h}$. (D) $\gamma_{\phi}^{p h}$ as a function of $\omega_{R}$. Comparison between the experimental values (magenta dots), values obtained by numerical integration of the system master equation (blue squares), and theoretical curves (magenta lines) given by Eq. [6 and using only measured parameters (the two lines limiting the grey area correspond to the lower and higher bounds of experimental uncertainties).

signature of the Quantum Zeno Effect. By fitting each Rabi curve with the analytical solution of Bloch equations [26] (using the measured $\Gamma_{1}^{-1}=225 \pm 10 \mathrm{~ns}$ ), we obtain in Fig. 7C the total decoherence rates $\Gamma_{2}\left(\omega_{R}, \bar{n}\right)$ that includes the measurement-induced dephasing $\Gamma_{\phi}^{p h}\left(\omega_{R}, \bar{n}\right)$ and the contribution $\Gamma_{2}\left(\omega_{R}, 0\right)=\Gamma_{\phi}^{0}\left(\omega_{R}\right)+\Gamma_{1} / 2$ from other dephasing sources and from energy relaxation $\left(\Gamma_{\phi}^{0}(2 \pi \times 5 \mathrm{MHz})=(810 \pm 20 \mathrm{~ns})^{-1}\right.$ in Fig. [7A for instance). For each $\omega_{R}$, the measured $\Gamma_{\phi}^{p h}\left(\omega_{R}, \bar{n}\right)$ are proportional to $\bar{n}$ as expected. Their slopes $\gamma_{\phi}^{p h}\left(\omega_{R}\right)$ 
determined by fitting $\Gamma_{\phi}^{p h}\left(\omega_{R}, \bar{n}\right)$ up to $\bar{n}=5$ are shown in Fig. $7 \mathrm{D}$ and are in good agreement with the predictions of Eq. [6] using the independendly measured values of $\chi$ and $\kappa$. We thus have a quantitative understanding of the measurement-induced dephasing in our system.

\section{E. Determination of the frequency response $R(\omega)$ of the measuring line}

The measuring line between the sample and the output signal includes several microwave circulators, filters, amplifiers, an IQ demodulator, two fast digitizers, and many sections of cables with connectors. Its frequency response $R(\omega)$ was measured in-situ in order to correct precisely the raw measured spectra $S(\omega)$. It is shown in supplementary Fig. 8 after normalization to 1 at zero frequency. The error bar shown $( \pm 1.5 \%)$ is an upper bound of the maximum systematic error over the whole frequency range.

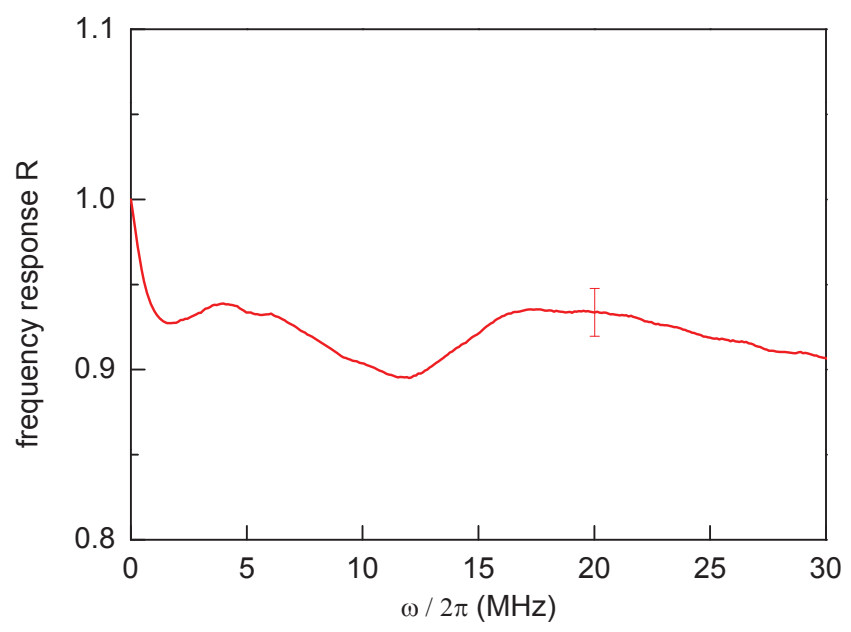

Figure 8: Frequency response $R(\omega)$ of the measuring line, including the amplification and demodulation chain. The error bar represent a constant maximum relative error.

\section{F. Calibration of the spectra : $\delta V(\bar{n})$}

According to the definition of $S(\omega)$, the conversion factor $\delta V(\bar{n}) / 2$ between the demodulated output signals in Volts and spin units is defined as $[\delta V(\bar{n})]^{2}=[\delta I(\bar{n})]^{2}+[\delta Q(\bar{n})]^{2}$, with $\delta X(\bar{n})$ the change in quadrature $X$ when the TLS state changes from $g$ to $e$. This definition has the great advantage of being insensitive to any drift or jitter of the relative microwave phase between the measurement source $V_{m}$ and the local oscillator used 


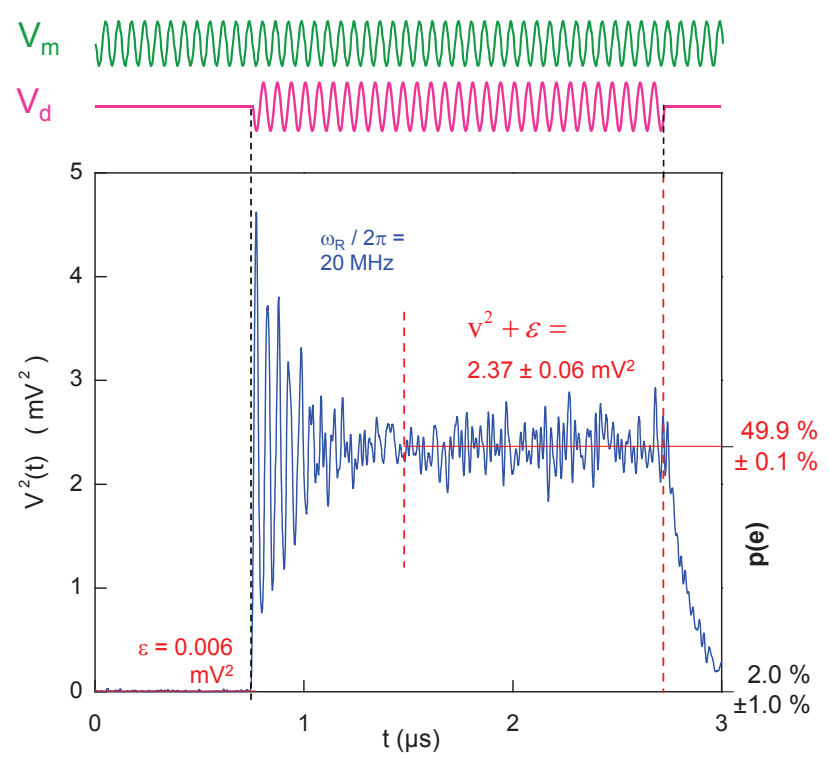

Figure 9: Calibration of the conversion factor $\delta V / 2$ between output demodulated voltage and spin units. A measurement pulse $V_{m}$ is applied with an amplitude corresponding to $\bar{n}=0.78$. After about one $\mu \mathrm{s}$, a saturating pulse is applied at $\omega_{\text {ge }}$ during about $2 \mu \mathrm{s}$. The $V^{2}(t)$ signal is measured and averaged over a few $10^{5}$ identical sequences. Starting from a thermal mixture of $98 \% g$ and $2 \% e$, the TLS undergoes Rabi oscillations at about $20 \mathrm{MHz}$ before it reaches its steady state with $50 \% g$ and $e$ population. The steady-state output yield $\delta V$ (see text).

for the demodulation. Since $(\delta V / 2)^{2}$ is the normalization factor of the spectrum used to test inequality (4), it is particularly important to determine it with the best possible precision. Although it could be calculated from several independently measured parameters, we found more accurate to calibrate it by direct measurement: we thus ensemble average $V^{2}(t)=\left[I_{O N}(t)-I_{O F F}(t)\right]^{2}+\left[Q_{O N}(t)-Q_{O F F}(t)\right]^{2}$ under saturation of the $g$-e transition (see supplementary Fig. 9), similarly to what is reported in B to determine $\chi$. In this experiment, $V_{m}$ is always $\mathrm{ON}$ and only $V_{d}$ is switched $\mathrm{ON}$ and $\mathrm{OFF}$, and $\omega_{R} / 2 \pi=20 \mathrm{MHz}$ so that $p(e)_{s t}=0.499 \pm 0.001 ; V^{2}(t)$ varies from $\epsilon=4<\xi_{0}^{2}>$ (with $<\xi_{0}^{2}>$ the variance of the noise on each $X$ up to $v^{2}+\varepsilon$ with $v=\left[p(e)_{s t}-p(e)_{0}\right] \delta V$ when saturation is reached. From supplementary Fig. 9, we obtain $(\delta V)^{2}=10.29 \pm 0.64 \mathrm{mV}^{2}$ for $\bar{n}=0.78$. It is important to note here that this calibration was done with the very same measurement power as that used to record the spectrum of Fig. 4, so that the uncertainty on $\bar{n}$ does not impact at all the violation of the Leggett-Garg inequality. Moreover, as averaging of this 
particular spectrum took about 13 hours, the calibration was performed twice, before and after averaging, in order to check that the experiment was stable: the second calibration yields $(\delta V)^{2}=10.59 \pm 0.64 \mathrm{mV}^{2}$. We thus take $(\delta V / 2)^{2}=2.61 \pm 0.16 \mathrm{mV}^{2}$ for the conversion factor in our test of inequality (4).

The experimental spectra of Fig. 3 obtained for different $\bar{n}$ have also been expressed in spin units by simply rescaling $\delta V(\bar{n}=0.78)$ by $\sqrt{\bar{n} / 0.78}$ at low $\bar{n}$. For $\bar{n}$ above 3 , we also take into account corrections to the dispersive approximation, which is valid only for $\bar{n} \ll n_{\text {crit }}=\Delta^{2} / 4 g_{0}^{2}=31$ [13] in our case (where $g_{0}$ is the TLS-resonator coupling constant). Using a model similar to [19], we keep the form of the dispersive Hamiltonian unchanged but use a modified dispersive constant $\chi(\bar{n})=\chi(0)(1-\lambda \bar{n})$ yielding a modified conversion factor $\delta V(\bar{n})(1-\lambda \bar{n})$. We determine $\lambda=7 \cdot 10^{-3}$ both experimentally and theoretically. Note that this correction gives noticeable effect only for $\bar{n}=15$ in Fig. $3 \mathrm{~F}$.

\section{G. Analytical formula for the frequency spectra}

The noise spectrum of a TLS undergoing coherent oscillations under continuous measurement has been computed in the case of a quantum dot coupled to a quantum point contact. Such a problem can be completely mapped onto our experiment with a transmon in a resonator with infinite bandwidth. In this limit, an exact analytical formula exists for the spectrum $S_{\hat{z}}(\omega)$ [9], which is simply the Fourier transform of the two-time correlation function $K_{\hat{z}}(\tau)=\left\langle\hat{\sigma}_{z}(t) \hat{\sigma}_{z}(t+\tau)\right\rangle_{t}$ obtained from the analytical solutions to the Bloch equations:

$$
\begin{aligned}
& S_{\hat{z}}(\omega)=\frac{4}{\left[\gamma^{2}+\left(\omega-\tilde{\omega}_{R}\right)^{2}\right]\left[\gamma^{2}+\right.}\left.\left(\omega+\tilde{\omega}_{R}\right)^{2}\right] \\
&\left.+\left[\left(1-z_{s t}^{2}\right)\left(\Gamma_{2}-\Gamma_{1}\right) / 2-\omega_{R}^{2} z_{s t}^{2} / \Gamma_{2}\right]\left(\gamma^{2}+\tilde{\omega}_{R}^{2}-\omega^{2}\right)\right\}, \\
&+
\end{aligned}
$$

where $\tilde{\omega}_{R}=\sqrt{\omega_{R}^{2}-\left(\Gamma_{2}-\Gamma_{1}\right)^{2} / 4}, \gamma=\left(\Gamma_{2}+\Gamma_{1}\right) / 2$, and $z_{s t}=-1 /\left(1+\Gamma_{1}^{-1} \Gamma_{2}^{-1} \omega_{R}^{2}\right)$ is the steady state solution. Due to the finite bandwidth of the detector, the measured signal $\tilde{S}_{z}$ is reduced as well as the dephasing rate according to Eq. (6). We modify Eq. 7 to take this into account by (i) multiplying $S_{\hat{z}}$ by the Lorentzian cutoff $C(\omega)=1 /\left(1+(2 \omega / \kappa)^{2}\right)$ and (ii) by changing the dephasing contribution $\Gamma_{\phi}$ to the total decoherence rate $\Gamma_{2}=\Gamma_{\phi}+\Gamma_{1} / 2$ into $\Gamma_{\phi} C(\omega)$; we obtain in this way an expression $\tilde{S}_{\hat{z}}(\omega)$. The validity of this phenomenological 
approach was checked by numerical simulations as explained in $\mathrm{H}$. The calculated spectra of Fig. 3 3 C-F and in the inset of Fig. 4 are obtained using the independently measured values $\Gamma_{1}=(200 \mathrm{~ns})^{-1}$ and $\Gamma_{\phi} C(\omega)=(150 \mathrm{~ns})^{-1}($ see $\mathrm{D})$.

\section{H. Numerical simulations}

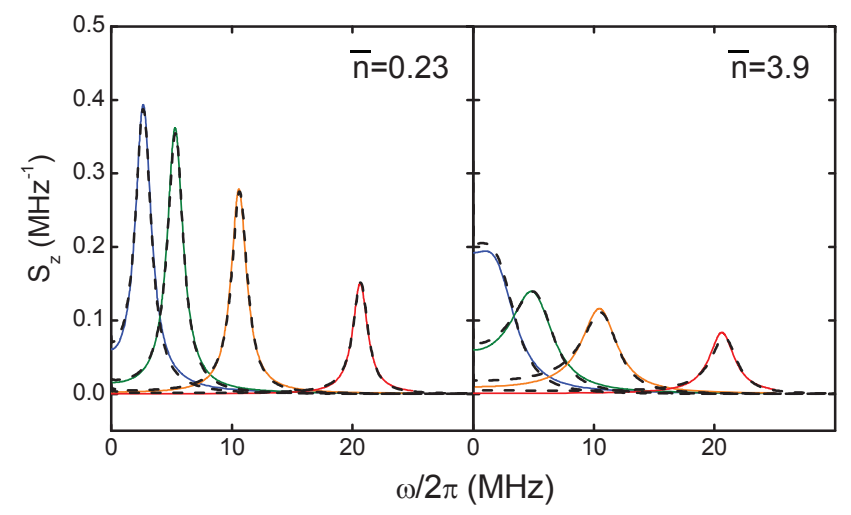

Figure 10: Comparison between the analytical spectra $\tilde{S}_{\hat{z}}$ (solid lines) and numerical simulations using the master equation as explained in the text (dashed black lines), for $\bar{n}=0.23$ and 3.9, and $\omega_{R} / 2 \pi=2.5,5,10,20 \mathrm{MHz}$. The agreeement is excellent.

We perform numerical calculations of the combined TLS-cavity field evolution by integrating the master equation of the system (as described in [19]). We model its coherent evolution with the effective dispersive Hamiltonian (valid in the dispersive limit $|\Delta| \gg g_{0}$ )

$$
H_{e f f} / \hbar=-\frac{\left(\omega_{g e}-\omega_{d}\right)}{2} \sigma_{z}+\left(\omega_{c}-\omega_{m}\right) a^{\dagger} a+\chi a^{\dagger} a \sigma_{z}+V_{m}\left(a^{\dagger}+a\right)+V_{d}\left(\sigma_{-}+\sigma_{+}\right)
$$

with $\omega_{d}$ and $\omega_{m}$ the frequencies of the sources $V_{d}$ and $V_{m}$ driving the TLS and the resonator. Taking into account the damping of the resonator field at rate $\kappa$ and of the TLS energy and coherence at rates $\Gamma_{1}$ and $\Gamma_{\phi}$, the master equation is

$$
\dot{\rho}=-\frac{i}{\hbar}\left[H_{e f f}, \rho(t)\right]+\kappa \mathcal{D}[a] \rho(t)+\Gamma_{1} \mathcal{D}\left[\sigma_{-}\right] \rho(t)+\Gamma_{\phi} \mathcal{D}\left[\sigma_{z}\right] \rho(t) / 2,
$$

with $\mathcal{D}[A] \rho=A \rho A^{\dagger}-A^{\dagger} A \rho / 2-\rho A^{\dagger} A / 2$. We integrated this equation using a quantum optics library available online [S1]. 
We used this numerical tool to perform simulations of Rabi oscillations dephased by $\bar{n}$ photons in the cavity (dark blue squares in supplementary Fig. 7 $7 \mathrm{~d}$ ), taking $\chi / 2 \pi=1.8 \mathrm{MHz}$. We also used it to compute the two-time correlation function of the detector output, i.e. $K^{\prime}(\tau)=\kappa\left\langle\operatorname{Re}\left[a^{\dagger}(t+\tau) a(t)\right]\right\rangle_{t}$, by using the quantum regression theorem [S2]; we then convert $K^{\prime}(\tau)$ into a power spectrum by Fast Fourier Transform. The conversion into spin units was done as in the experiment, by calculating the output signal at saturation $\delta V$ of a continuously monitored Rabi oscillation. We were able to perform the simulations only up to $\bar{n}=7.8$, yielding the dashed lines shown in Fig. $3 \mathrm{~B} \mathrm{C}-\mathrm{F}$. The agreement with the analytical formula is excellent for all calculated curves, as can be seen in Fig. 10.

\section{Experimental uncertainties in the determination of $f_{L G}(\tau)$}

As mentionned in the text, the experimental points $f_{L G}(\tau)=2 K(\tau)-K(2 \tau)$ of Fig. 4 are obtained from the inverse Fourier transform $K(\tau)$ of $S_{z}(\omega)=\tilde{S}_{z}(\omega) / C(\omega)$ with

$$
\tilde{S}_{z}(\omega)=\frac{S_{O N}(\omega)-S_{O F F}(\omega)}{R(\omega)(\delta V / 2)^{2}},
$$

with $R(0)=C(0)=1$ and $\delta V / 2$ being measured at zero frequency. The systematic error bars on $f_{L G}(\tau)$ (in green in Fig. (4) result from the sum of the three maximum relative uncertainties, $\Delta R / R= \pm 1.5 \%, \Delta(\delta V / 2)^{2} /(\delta V / 2)^{2}= \pm 6.1 \%$ and $\Delta C / C=2(\Delta \kappa / \kappa) /\left[1+(\kappa / 2 \omega)^{2}\right]$ with $\Delta \kappa / \kappa= \pm 2.6 \%$. Note that the frequency dependent error $\Delta C / C$ is propagated exactly through the calculation of $f_{L G}(\tau)$ and contributes to $\Delta f_{L G} / f_{L G}$ by $\pm 0.8 \%$ where inequation (4) is violated. The total systematic error at that point is thus $\pm 8.4 \%$. The statistical standard deviation on each $f_{L G}(\tau)$ data point is computed by propagating the statistical error on the measured Rabi spectrum (i) through the division by the cavity filtering $C(\omega)$, (ii) through the definition of the inverse Fourier transform, and (iii) through the difference $2 K(\tau)-K(2 \tau)$. Each point $k=1$ to $N$ of the $S_{z}(\omega)$ spectrum with bin size $\Delta f=100 \mathrm{kHz}$ has a constant standard deviation $\sigma_{0}$ measured in the $22-30 \mathrm{MHz}$ region where the spectral density is zero. Consequently, the standard deviation on each point $k$ of the corrected spectrum is $\sigma_{k}=\sigma_{0} / C[2 \pi \Delta f(k-1)]$. Finally, the standard deviation $\sigma_{r}$ on each point $r$ of $f_{L G}[\tau=(r-1) /(N \Delta f)]$ is 


$$
\sigma_{r}=\Delta f \sqrt{\sigma_{k=1}^{2}+4 \sum_{k=2}^{N / 2} \sigma_{k}^{2}\left[2 \cos \frac{2 \pi(r-1)(k-1)}{N}-\cos \frac{2 \pi 2(r-1)(k-1)}{N}\right]^{2}} .
$$

A conventional $2 \sigma_{r}$ statistical contribution is added to the systematic error to form the total red error bars of Fig. 4. At the second point $\tau=17 \mathrm{~ns}$ where inequation (4) is violated, the standard deviation $s \equiv \sigma_{2}=0.065$, and the bottom of the systematic error bar is $4.9 \mathrm{~s}$ above 1 .

[S1] Tan, S.M. A Computational Toolbox for Quantum and Atomic Optics, available online http://www.qo.phy.auckland.ac.nz/qotoolbox.html

[S2] Walls, D.F. \& Milburn, G. Quantum Optics, (Springer ed., Heidelberg 2008), chap. 6.

[S3] Ithier, G. et al. Decoherence in a superconducting quantum bit circuit. Phys. Rev. B 72, 134519 (2005). 